\title{
High-throughput, non-invasive prenatal testing for fetal rhesus D status in RhD- negative women: a systematic review and meta-analysis
}

Huiqin Yang ${ }^{1 *}$, Alexis Llewellyn², Ruth Walker², Melissa Harden², Pedro Saramago ${ }^{3}$, Susan Griffin ${ }^{3}$ and Mark Simmonds ${ }^{2}$

\begin{abstract}
Background: High-throughput non-invasive prenatal testing (NIPT) for fetal Rhesus D (RhD) status could avoid unnecessary treatment with anti-D immunoglobulin for RhD-negative women found to be carrying an RhD-negative fetus. We aimed to assess the diagnostic accuracy of high-throughput NIPT for fetal RhD status in RhD-negative women not known to be sensitized to the RhD antigen, by performing a systematic review and meta-analysis.

Methods: Prospective cohort studies of high-throughput NIPT used to determine fetal RhD status were included. The eligible population were pregnant women who were RhD negative and not known to be sensitized to RhD antigen. The index test was high-throughput, NIPT cell-free fetal DNA tests of maternal plasma used to determine fetal RhD status. The reference standard considered was serologic cord blood testing at birth. Databases including MEDLINE, EMBASE, and Science Citation Index were searched up to February 2016.

Two reviewers independently screened titles and abstracts and assessed full texts identified as potentially relevant. Risk of bias was assessed using QUADAS-2. The bivariate and hierarchical summary receiver-operating characteristic (HSROC) models were fitted to calculate summary estimates of sensitivity, specificity, false positive and false negative rates, and the associated 95\% confidence intervals (Cls).

Results: A total of 3921 references records were identified through electronic searches. Eight studies were included in the systematic review. Six studies were judged to be at low risk of bias. The HSROC models demonstrated high diagnostic performance of high-throughput NIPT testing for women tested at or after 11 weeks gestation. In the primary analysis for diagnostic accuracy, women with an inconclusive test result were treated as having tested positive. The false negative rate (incorrectly classed as RhD negative) was $0.34 \%(95 \% \mathrm{Cl} 0.15$ to 0.76 ) and the false positive rate (incorrectly classed as RhD positive) was 3.86\% (95\% Cl 2.54 to 5.82). There was limited evidence for non-white women and multiple pregnancies.
\end{abstract}

Conclusions: High-throughput NIPT is sufficiently accurate to detect fetal RhD status in RhD-negative women and would considerably reduce unnecessary treatment with routine anti-D immunoglobulin. The applicability of these findings to non-white women and women with multiple pregnancies is uncertain.

Keywords: Fetal rhesus D status, Non-invasive prenatal testing, Diagnostic accuracy, Anti-D immunoglobulin, Systematic review

\footnotetext{
* Correspondence: H.Yang@exeter.ac.uk

'University of Exeter Medical School, St Luke's Campus, Heavitree Road,

Exeter EX1 2LU, UK

Full list of author information is available at the end of the article
}

(c) The Author(s). 2019 Open Access This article is distributed under the terms of the Creative Commons Attribution 4.0 International License (http://creativecommons.org/licenses/by/4.0/), which permits unrestricted use, distribution, and reproduction in any medium, provided you give appropriate credit to the original author(s) and the source, provide a link to the Creative Commons license, and indicate if changes were made. The Creative Commons Public Domain Dedication waiver (http://creativecommons.org/publicdomain/zero/1.0/) applies to the data made available in this article, unless otherwise stated. 


\section{Introduction}

Pregnant women who have an $\mathrm{RhD}$-negative blood type may carry an $\mathrm{RhD}$-positive fetus. The presence of fetal $\mathrm{RhD}$-positive cells in the maternal circulation can cause a mother who is $\mathrm{RhD}$ negative to produce anti-D antibodies against the $\mathrm{RhD}$ antigen. This immune response, termed sensitisation, can happen at any time during the pregnancy, but it is most common in the third trimester and during childbirth [1].

The process of sensitisation itself has no adverse effects on the mother and does not usually affect the pregnancy during which it occurs. However, in a subsequent pregnancy with an $\mathrm{RhD}$-positive fetus in women who have been sensitized to the RhD antigen, the woman's anti-D antibodies may cross the placenta resulting in haemolytic disease of the fetus and newborn.

This can cause severe fetal anemia that leads to fetal heart failure, fluid retention and swelling (hydrops), hyperbilirubinaemia, kernicterus, and perinatal death [2].

Prophylaxis with anti-RhD immunoglobulin can substantially reduce the risk of sensitisation in $\mathrm{RhD}$-negative women and hence the prevalence of haemolytic disease of the fetus and newborn [3]. The introduction of routine antenatal prophylaxis during the third trimester of pregnancy has led to a reduction in sensitisation, resulting in a decrease in mortality associated with haemolytic disease of the fetus and newborn from 46 in 100,000 births before 1969 to 1.6 in 100,000 births by 1991 [4].

Currently, the National Institute for Health and Clinical Excellence (NICE) guideline on antenatal care recommends that women should be offered testing for blood group and rhesus D status in early pregnancy [5]. In those identified as $\mathrm{RhD}$ negative and without preformed antibodies, administration of anti-D immunoglobulin is recommended both as prophylaxis and following potential sensitizing events to prevent the sensitisation from occurring. Routine antenatal prophylaxis with anti-D immunoglobulin can be given as two doses at weeks 28 and 34 of pregnancy or as a single dose between 28 and 30 weeks [5].

Non-invasive prenatal testing (NIPT) of fetal RhD status uses a real-time quantitative polymerase chain reaction (PCR) method to detect cell-free fetal DNA-small fragments of extracellular DNA shed from the placenta circulating freely in the maternal plasma. High-throughput NIPT performs the test using an automated platform capable of performing a large number of tests simultaneously, and is therefore suitable for large-scale population screening of pregnant women. High-throughput NIPT for fetal $\mathrm{RhD}$ status may enable anti-D immunoglobulin to be withheld from RhD-negative women who are predicted to be carrying an $\mathrm{RhD}$-negative fetus. Pregnant women found to be carrying an RhD-negative fetus could avoid unnecessary treatment with anti-D immunoglobulin (which is a human polyclonal plasma-derived product), along with the potential risk associated with administration of blood products. In addition, these women may not need the provision of anti-D immunoglobulin following potentially sensitizing events, and there may no longer be a need for serologic cord testing at birth. High-throughput NIPT is already used in this way in some European countries $[6,7]$.

However, the diagnostic accuracy of high-throughput NIPT for fetal Rhesus D status in RhD-negative women not known to be sensitized to the RhD antigen is uncertain. The National Institute of Health Research Health Technology Assessment programme commissioned a systematic review and economic evaluation to assess the diagnostic accuracy and cost-effectiveness of high-throughput NIPT for the detection of fetal Rhesus D status in $\mathrm{RhD}$-negative women. This systematic review formed part of a larger report on high-throughput NIPT which also considered broader issues in its clinical value and implementation and a full economic analysis [8]. This work was used to inform the recent NICE guideline regarding the recommendation of high-throughput NIPT for fetal Rhesus D status [9].

\section{Methods}

The complete methodology of the original wider review is reported elsewhere [8]. This section summarizes the methodology of the diagnostic accuracy review.

\section{Data sources and searches}

We conducted a systematic review following the PRISMA statement [10] and registered the review on PROSPERO. The following databases were searched for relevant studies from inception to November 2015: MEDLINE, MEDLINE In-Process, CINAHL, Cochrane Central Register of Controlled Trials (CENTRAL), Cochrane Database of Systematic Reviews (CDSR), Database of Abstracts of Reviews of Effects (DARE), EMBASE, Health Technology Assessment (HTA) database, Maternity and Infant Care, PubMed, and the Science Citation Index. In addition, the following resources were searched for on-going, unpublished, or gray literature: ClinicalTrials.gov, Conference Proceedings Citation Index: Science, EU Clinical Trials Register, PROSPERO, and the WHO International Clinical Trials Registry Platform portal. An updated search was performed in February 2016.

Both published and unpublished literature were identified from systematic searches of electronic sources, consultation with experts in the field, and reference checking of relevant systematic reviews and included studies. Search strategies were developed by an information specialist $(\mathrm{MH})$. The search strategy can be found in Additional file 1. 


\section{Study selection}

Prospective cohort studies of high-throughput NIPT used to determine fetal $\mathrm{RhD}$ status were eligible for inclusion. We considered as high-throughput, any NIPT tests which were conducted using an automated robotic platform (including automated DNA extraction and liquid handling) and were able to process large numbers of samples rapidly for large-scale screening purposes. Studies where this test was used for diagnosis (rather than screening) of sensitized women were excluded.

The inclusion criteria for population, index test, reference standard, and outcomes are listed below:

- The eligible population were pregnant women who were $\mathrm{RhD}$ negative and not known to be sensitized to $\mathrm{RhD}$ antigen.

- The index test was high-throughput, NIPT cell-free fetal DNA tests of maternal plasma used to determine fetal RhD status.

- The reference standard considered was serologic cord blood testing at birth.

- The eligible studies had to report diagnostic accuracy data such as absolute numbers of true positive, false positive, true negative, and false negative test results.

Two researchers independently screened the titles and abstracts of all reports identified by the search strategy, and full-text papers were subsequently obtained for assessment. Only reports published in English were sought. There were no restrictions for publication date. Full-text copies of all studies deemed to be potentially relevant were obtained and two reviewers independently assessed them for inclusion (HY, AL). Any disagreements were resolved by consensus or by a third reviewer (RW).

\section{Data extraction}

We selected the most recent or most complete report in cases of multiple reports for a given study or when we could not exclude the possibility of overlapping populations. One reviewer independently extracted details from full-text studies including study design, participants, index, comparator and reference standard tests, and outcome data. The data extraction was checked by another reviewer. Any disagreements were resolved by consensus (between $\mathrm{HY}$ and $\mathrm{AL}$ ) or with a third reviewer (RW).

We extracted the number of true positives, true negatives, false positives, and false negatives for each index test evaluated in each study in order to construct $2 \times 2$ tables. If reported, we extracted data on the number of undetermined or uninterpretable results. Study authors were contacted if some data were unclear or missing.

\section{Quality assessment}

Risk of bias was assessed using a modified version of the quality assessment of diagnostic accuracy studies (QUADAS-2) checklist [11]. The QUADAS-2 tool consists of four key domains: (1) patient selection, (2) index test, (3) reference standard, and (4) flow of patients through the study and timing of the index test(s) and reference standard. Each domain was assessed in terms of the risk of bias. The first three domains were also assessed for concerns regarding their applicability in terms of whether (1) the participants and setting, (2) the index test, its conduct or interpretation, and (3) the target condition as defined by the reference standard were applicable to the UK context. One reviewer (AL) independently assessed the quality of all included studies in terms of risk of bias. The quality assessment was checked by another reviewer (HY). Any disagreements were resolved by consensus or by a third party (RW).

\section{Data synthesis}

For diagnostic accuracy outcomes, estimates of sensitivity, specificity, and false positive and false negative rates were calculated and presented on forest plots and in receiver-operating characteristic (ROC) space to assess the heterogeneity in test accuracy within and between studies. The hierarchical bivariate model [12] was fitted to calculate summary estimates of sensitivity, specificity, and false positive and false negative rates and the associated $95 \%$ confidence intervals (CIs). The hierarchical summary ROC (HSROC) model [13] was fitted to produce summary ROC curves. Both models jointly model sensitivity and specificity and account for the correlation between them. Heterogeneity in sensitivity and specificity was also assessed using the $I^{2}$ statistic. All analyses were performed using $\mathrm{R}$ software [14, 15]. Because NIPT testing is highly accurate, we present the results in terms of the false positive rate (FPR) (incorrectly testing positive and being offered unnecessary anti-D prophylaxis) and false negative rate (FNR) (incorrectly testing negative; at risk of sensitisation as women do not receive anti-D prophylaxis), rather than the conventional sensitivity and specificity.

Some NIPT results are inconclusive and unable to predict the RhD status of the fetus. Current UK practice is to treat such test results as if they predicted an $\mathrm{RhD}$-positive fetus, and this approach was used in the primary analysis of diagnostic accuracy. Sensitivity analyses were conducted to explore the robustness of the results by including and excluding such inconclusive test results. A further sensitivity analysis included only UK (Bristol)-based studies, as this review was intended to inform UK practice. Furthermore, as test accuracy may vary according to the gestation age when NIPT is performed, we investigated the impact of test timing by 
plotting diagnostic accuracy against time, and performing meta-regressions against test timing. No analysis for small study effects or publication bias was performed because there were too few studies identified to justify this.

\section{Results}

The literature searches of bibliographic databases identified 3921 references. After initial screening of titles and abstracts, 227 were considered to be potentially relevant and were ordered for full paper screening. In total, eight studies $[6,7,16-19]$ were included in the diagnostic review of high-throughput NIPT testing. Six studies reported inconclusive results. Figure 1 shows a flow diagram outlining the screening process with reasons for exclusion of full-text papers.

Table 1 presents the summary information of characteristics of the included diagnostic accuracy studies. All the studies were prospective studies and conducted in European countries. Four studies were conducted in England [16-19], three of which were based at Bristol [16-18]. The sample size of studies ranged from 282 to 18,383 . Most studies recruited pregnant women with a gestational age of 10 to 28 weeks. Most participants were white, and most pregnancies were singleton. All studies used maternal plasma as their sample source. A robotic DNA extraction instrument was employed in all studies. The studies used a number of robotic platforms including MDx BioRobot, MagNa Pure 96, MagNA Pure LC, and COBAS AmpliPrep. For PCR, all studies targeted at least two exons (generally exons 5 and 7) and at least two controls for $R H D$ assay ( $\mathrm{RhD}$-positive $\mathrm{DNA}$ and RhD-negative DNA) except for the study by Wikman et al. [20] which targeted exon 4 only and used GAPDH DNA as control. The reference standard used in all studies was cord blood serology, except for Akolekar et al. [17] which did not describe its reference standard. Where reported, rates of inconclusive results ranged from $1.0 \%$ [20] to $14.3 \%$ [19].

\section{Risk of bias}

Table 2 presents a summary of the results for the risk of bias assessment. The majority of included studies were judged to be at low risk of bias, but two studies $[7,19]$ were judged to be at high risk of bias. The study by Akolekar et al. [19] reported that the targeted RhDnegative women were selected from a database, but it was unclear whether this selection was conducted on a random basis. The study enrolled a large proportion of Africans (19.3\%) which may not be representative of the general population of pregnant women in the UK. This may have contributed to the larger than average proportion of inconclusive results (14.3\%). Characteristics of the reference standard were also poorly reported in this study. In the study by Thurik et al. [7], only $80 \%$ of participants received a reference standard. The reasons why cord blood serology was not conducted in a significant proportion of the study population were not stated. This study also reported that its prediction algorithm was judged daily and modified as needed, which may have introduced bias in the diagnostic accuracy estimates.

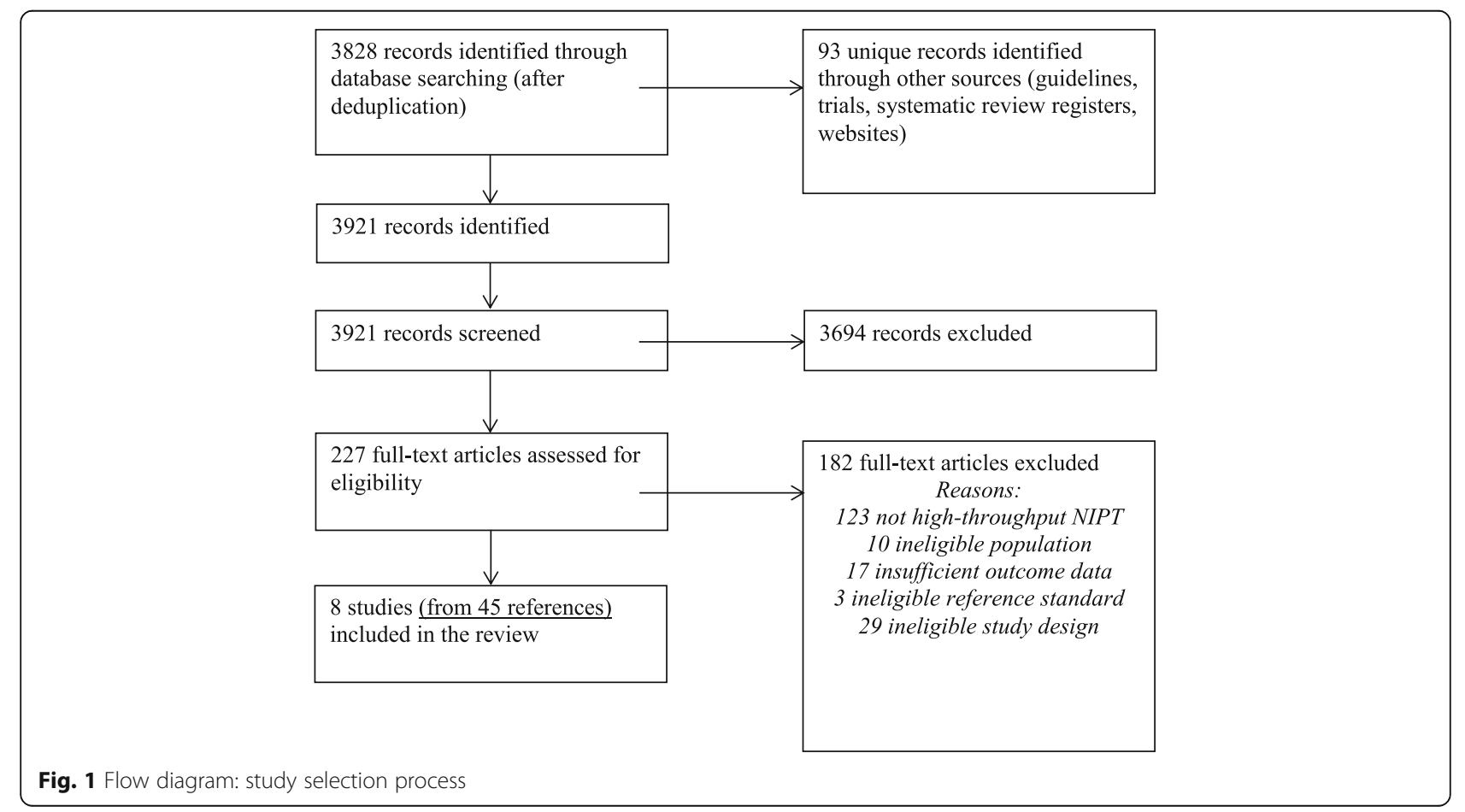




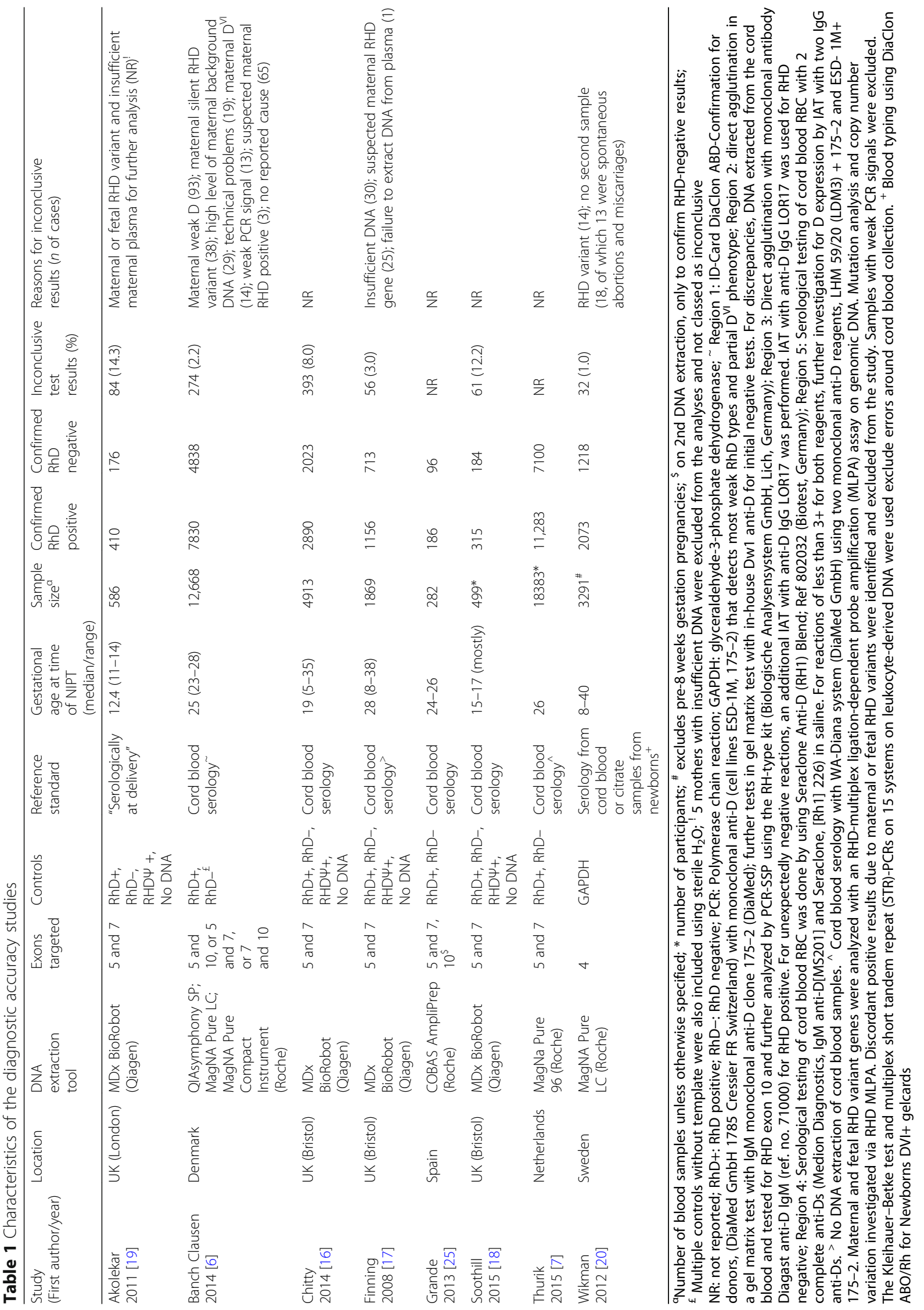


Table 2 Risk of bias of included studies

\begin{tabular}{|c|c|c|c|c|c|c|c|}
\hline \multirow[t]{2}{*}{ Study } & \multicolumn{4}{|l|}{ Risk of bias } & \multicolumn{3}{|c|}{ Applicability concerns } \\
\hline & Patient selection & Index test & Reference standard & Flow and timing & Patient selection & Index test & Reference standard \\
\hline Akolekar (2011) [19] & High & High & Unclear & Unclear & High & Low & Unclear \\
\hline Banch-Clausen (2014) [6] & Low & Low & Low & Low & Unclear & Low & Low \\
\hline Chitty (2014) [16] & Low & Low & Low & Low & Low & Low & Low \\
\hline Finning (2008) [17] & Low & Low & Low & Low & Low & Low & Low \\
\hline Grande (2013) [25] & Low & Low & Low & Low & Low & Low & Low \\
\hline Soothill (2015) [18] & Low & Unclear & Low & Low & Low & Low & Low \\
\hline Thurik (2015) [7] & Low & High & Low & High & Low & Low & Low \\
\hline Wikman (2012) [20] & Low & Low & Low & Low & Unclear & High & Low \\
\hline
\end{tabular}

High: high risk of bias; Low: low risk of bias

NIPT as an automated procedure was deemed to be of limited risk to human error, and multiple controls were used for RHD assays in all except one study [20]. The index test of NIPT was conducted independent of the reference standard, and the results of one were considered unlikely to influence the results of the other; therefore, the risk of incorporation bias was considered low. It appears that most studies prospectively recruited consecutive samples from clinical practice. Only three studies stated that their diagnostic threshold was pre-specified during the conduct of the screening program $[6,16,17]$.

The results of the studies were considered broadly applicable to the use of high-throughput NIPT for nationwide screening purposes, except for two studies $[19,20]$. In particular, the NIPT test used in the study by Wikman et al. [21] only targeted exon 4, unlike all other included studies where at least two exons $(5,7$, and/or $10)$ were targeted. It is generally accepted that a combination such as of exons 5 and 7 should be targeted to discriminate the pseudogene $R H D \Psi$, particularly present in individuals of African origin [22].

\section{Meta-analysis}

The results of the bivariate meta-analyses are shown in Table 3. These show that NIPT is a highly accurate test. The false negative rate (where women would not be offered anti-D prophylaxis and so be at risk of sensitisation) is very low at $0.34 \%$ (95\% CI 0.15 to 0.76 ). When treating women with an inconclusive test result as if they were positive, the false positive rate is $3.86 \%$ (95\% CI 2.54 to 5.82). Excluding inconclusive test results reduces this to $1.26 \%$ (95\% CI 0.87 to 1.83 ). Therefore, most false positive results occur in women with inconclusive test results.

There was some evidence of inconsistency across studies. $I^{2}$ was $75 \%$ for the false negative rate and $99 \%$ for the false positive rate. It should be noted that these high heterogeneities are, in part, a consequence of the high accuracy of the test and the large size of the studies (and consequent small within-study variance, because $I^{2}$ increases as the average within-study variance declines). They do not necessarily indicate any clinically meaningful differences between studies. The heterogeneity in false positive rates is likely to be a consequence of differing reporting and handling of inconclusive tests.

Studies conducted in Bristol had a lower false negative rate $(0.21 \%, 95 \% \mathrm{CI} 0.09$ to 0.48$)$, with a consequently higher false positive rate (5.73\%, $95 \%$ CI 4.58 to 7.16$)$. This suggests that the Bristol high-throughput NIPT testing approach, in which the MDx Bio Robot machine is used, may be using a different test threshold to other countries, which further minimizes false negative findings.

Figure 2 shows the results of the bivariate and the summary HSROC curve for this primary analysis, which is presented in terms of false positive and false negative rates. The black circle is the summary effect estimate from a bivariate model, and the black curve is the HSROC curve. This plot shows that the studies were generally consistent in terms of false negative results, except for two outlying studies $[19,20]$. The study by Wikman et al. [20] conducted most NIPT tests in the first trimester, earlier

Table 3 Bivariate meta-analyses of false positive and negative rates

\begin{tabular}{|c|c|c|c|c|c|}
\hline \multirow[t]{2}{*}{ Analysis case } & \multirow{2}{*}{$\begin{array}{l}\text { Number of } \\
\text { studies }\end{array}$} & \multicolumn{2}{|c|}{ False negative rate (at risk of sensitisation) } & \multicolumn{2}{|c|}{ False positive rate (unnecessary anti-D) } \\
\hline & & Estimate (\%) & $95 \% \mathrm{Cl}$ & Estimate $(\%)$ & $95 \% \mathrm{Cl}$ \\
\hline Inconclusive tests treated as test positive & 8 & 0.34 & $0.15-0.76$ & 3.86 & $2.54-5.82$ \\
\hline Excluding all women with inconclusive test results & 8 & 0.35 & $0.15-0.82$ & 1.26 & $0.87-1.83$ \\
\hline Studies conducted in Bristol only & 3 & 0.21 & $0.09-0.48$ & 5.73 & $4.58-7.16$ \\
\hline
\end{tabular}




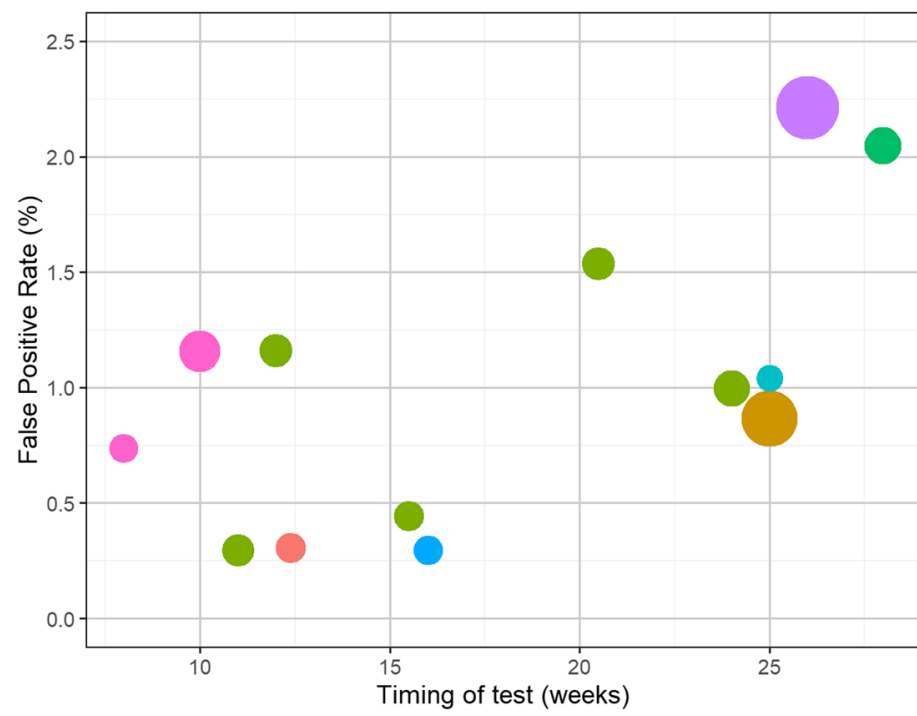

Study

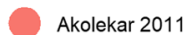

Banch Clausen 2014

Chitty 2014

Finning 2008

Grande 2013

Soothill 2015

Thurik 2015

Wikman 2012

Fig. $2 \mathrm{HSROC}$ and bivariate meta-analysis

than other studies. The studies are less consistent in false positive rates. This is most likely because the studies have different numbers of inconclusive test results, and different methods of handling such results.

\section{Timing of NIPT tests}

Figure 3 shows the false negative rates plotted by gestational age at time of high-throughput NIPT testing. It suggests that false negative rates after the first trimester (i.e., after around 13 weeks' gestation) were consistent, irrespective of timing, but false negative rates were higher in the first trimester. This pattern is most visible in the Chitty study [16] which reported diagnostic accuracy at a range of test timings. Given the limited amount of data, no formal statistical test could be performed to confirm this conclusion. Additional file 2: Figure $\mathrm{S} 1$ shows the false positive rates plotted by gestational age at time of high-throughput NIPT testing. There was no obvious pattern from this figure.

We also evaluated the impact of the timing of high-throughput NIPT testing on the number of inconclusive test results. As seen in Additional file 3: Figure S2, there is a suggestion that the percentage of inconclusive results for this test reduces as the gestational age increases. This is most obvious in the study by Chitty et al. [16].

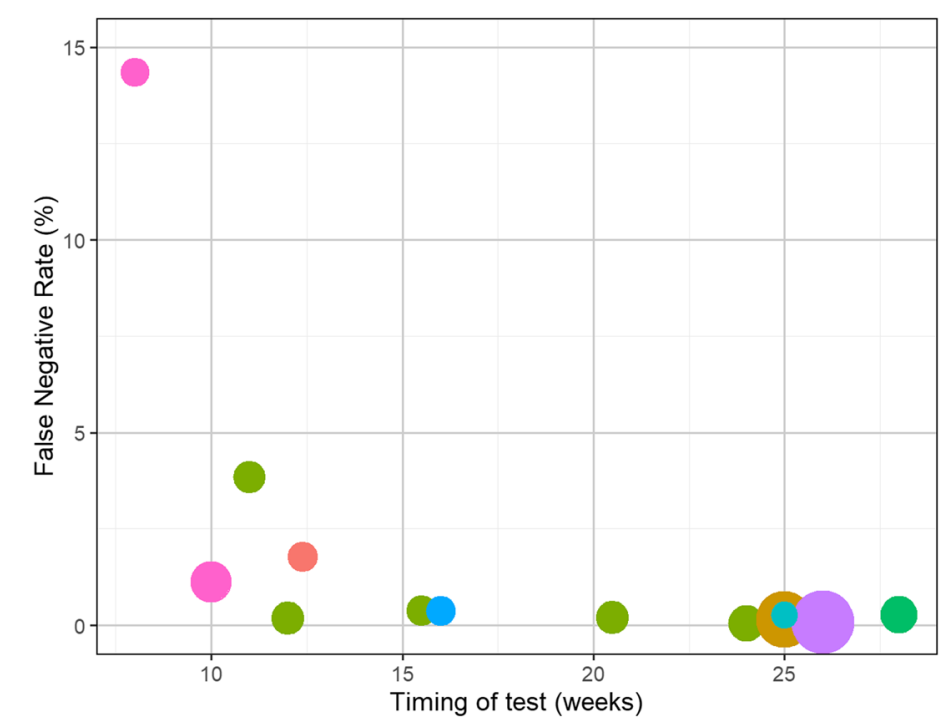

Study

Akolekar 2011

Banch Clausen 2014

Chitty 2014

Finning 2008

Grande 2013

Soothill 2015

Thurik 2015

Wikman 2012

Fig. 3 False negative rate by gestational age at time of NIPT 


\section{Impact on UK practice}

We considered the likely impact of implementing NIPT to guide anti-D prophylaxis in the UK by conducting a simulation study, simulating a representation of the UK population using data sourced from the review (see Additional file 4 for input parameters).

Based on the results of the three Bristol-based studies, we assumed that $60.7 \%$ of $\mathrm{RhD}$-negative women have an $\mathrm{RhD}$-positive fetus, and $6.7 \%$ of women have an inconclusive NIPT result. Given this, the results of the diagnostic meta-analysis, and parameters described in Additional file 4, using NIPT would result in anti-D prophylaxis being received by $65.9 \%$ of $\mathrm{RhD}$-negative women. It would reduce the numbers of women receiving unnecessary anti-D from 38.9 to $5.7 \%$. The number of women who miss out on potentially beneficial anti-D would rise from 0.6 to $1.2 \%$, leading to potentially more sensitisations: an extra 3 per 100,000 women if postpartum cord blood testing continues, or 13 per 100,000 if it is withdrawn. Sensitisation rates using universal anti-D administration were estimated to be 280 per 100,000 women, so this increase is small.

It would however mean $0.12 \%$ of women with an $\mathrm{RhD}$-positive fetus would not be offered anti-D and so would be at risk of sensitisation.

\section{Discussion}

In this systematic review, we identified eight studies that evaluated diagnostic accuracy of high-throughput NIPT. Six of these studies were judged to be at low risk of bias. Meta-analyses showed very high diagnostic accuracy of high-throughput NIPT testing.

Diagnostic accuracy of high-throughput NIPT varied by gestational age. The data suggest that highthroughput NIPT testing is insufficiently accurate in the first trimester, but is consistently accurate at any time thereafter. This might be due to low concentration of cell-free fetal DNA in early pregnancy [23] but an increased concentration of cell-free fetal DNA after the end of the first trimester [24].

Given the very high diagnostic accuracy performance of high-throughput NIPT testing, implementing highthroughput NIPT for fetal RhD screening in all RhDnegative women nationwide could be feasible. The results suggest it would substantially reduce the need for antenatal anti-D prophylaxis, while only marginally increasing the risk of sensitisation due to false negative test results. NIPT testing could be conducted, with low false positive rates, at any time from the second trimester onwards, perhaps to coincide with routine antenatal blood tests. Any nationwide NIPT screening program will require careful logistical management to ensure that blood samples are transported to laboratories and tested quickly and that results are reliably returned to general practitioners and midwives.

\section{Limitations}

We performed extensive literature searches with an attempt to maximize retrieval of potentially relevant studies. These included electronic searches of a variety of bibliographic databases as well as screening of clinical trial registers and conference proceedings to identify unpublished studies. However, only studies in English were included; therefore, some potentially relevant non-English language studies may have been missed. There was some evidence of inconsistency in the meta-analysis of diagnostic accuracy studies. The observed heterogeneity may be due to variations in methods used in the high-throughput NIPT approach (e.g., different diagnostic accuracy thresholds used, and different number and types of exons targeted, gestational age at the time of testing, and different methods of handling inconclusive test results). In addition, there was variation in the reporting of included studies. Particularly, two studies [7, 25] did not report the number of inconclusive results of the test and some studies did not report detailed reasons for inconclusive results. The simulation study assumes that the input probabilities are accurate and does not account for any uncertainty in their estimation. Therefore, results of the simulation study should be considered to be illustrative only and not definitive estimates of effect.

\section{Implications for future research}

Further large prospective cohort studies evaluating diagnostic accuracy of high-throughput NIPT in women of non-white ethnicity are required. This is of particular concern as non-white women are more likely to have less accurate test results. For example, in people with African ethnicity, because of the presence of $R H D$ pseudogene [26], prenatal detection of fetal $\mathrm{RhD}$ type from maternal blood would lead to higher rates of false positive results in this particular population. Further research to improve the NIPT test itself is also warranted, especially for reducing the number of inconclusive test results.

\section{Conclusions}

The findings from this systematic review have demonstrated high diagnostic performance of high-throughput NIPT testing for the detection of fetal $\mathrm{RhD}$ status in $\mathrm{RhD}$-negative women, with very low false positive and false negative rates in women tested at or after 11 weeks' gestation. The use of high-throughput NIPT testing as a routine screening test for fetal $\mathrm{RhD}$ status in $\mathrm{RhD}$-negative women can largely remove unnecessary exposure to prophylactic anti-D treatment. Due to limited evidence, the accuracy of NIPT in non-white women and multiple pregnancies is unclear. 


\section{Additional files}

Additional file 1: Search strategy. (DOCX $15 \mathrm{~kb}$ )

Additional file 2: Figure S1. False positive rate by gestational age at time of NIPT. (TIFF $241 \mathrm{~kb}$ )

Additional file 3: Figure S2. Inconclusive results by test timing (TIFF $67 \mathrm{~kb}$ )

Additional file 4: Probability estimates derived from published data, used in the simulation study. (DOCX $15 \mathrm{~kb}$ )

\section{Acknowledgements}

We would like to thank the following for providing advice on the original project: Dr. Finning Kirstin, NHS Blood and Transplant; Professor Peter Soothill, Emeritus Professor at the University of Bristol; Professor Lyn Chitty, Institute of Child Health, University College London.

This project was funded by the UK National Institute for Health Research (NIHR) health technology assessment programme (project number 15/17/02) The views and opinions expressed by authors in this publication are those of the authors and do not necessarily reflect those of the NIHR health technology assessment programme of the Department of Health.

\section{Funding}

This research was funded by the National Institute of Health Research as part of the National Institute for Health and Care Excellence diagnostics assessment (DAR) programme. The funders were not involved in any part of the review process, or in the writing of this paper. PROSPERO registration number: CRD42015029497.

\section{Availability of data and materials}

The datasets generated during and/or analyzed during the current study are available from the corresponding author on reasonable request.

\section{Authors' contributions}

$H Y, M S$, and AL developed the protocol with assistance from PS, SG, and MH. Study selection, data extraction, and quality assessment were conducted by HY, AL, and RW. Analysis was conducted by MS. Literature searches were designed and conducted by $\mathrm{MH}$. HY interpreted the evidence and was responsible for writing the manuscript. All authors contributed to the editing of this manuscript. All authors read and approved the final manuscript.

\section{Ethics approval and consent to participate}

No human subjects, human material, or human data were involved in this research, which is based on literature review.

\section{Consent for publication}

Not applicable

\section{Competing interests}

The authors declare that they have no competing interests.

\section{Publisher's Note}

Springer Nature remains neutral with regard to jurisdictional claims in published maps and institutional affiliations.

\section{Author details}

'University of Exeter Medical School, St Luke's Campus, Heavitree Road, Exeter EX1 2LU, UK. ${ }^{2}$ Centre for Reviews and Dissemination, University of York, York YO10 5DD, UK. ${ }^{3}$ Centre for Health Economics, University of York, York YO10 5DD, UK.

Received: 23 November 2017 Accepted: 8 January 2019 Published online: 14 February 2019

\section{References}

1. Urbaniak SJ, Greiss MA. RhD haemolytic disease of the fetus and the newborn. Blood Rev. 2000;14(1):44-61.

2. Murray NA, Roberts IA. Haemolytic disease of the newborn. Arch Dis Child Fetal Neonatal Ed. 2007;92(2):F83-8.
3. Kumar S, Regan F. Management of pregnancies with RhD alloimmunisation. BMJ. 2005:330(7502):1255-8. https://doi.org/10.1136/bmj.330.7502.1255.

4. Pilgrim H, Lloyd-Jones MRA. Routine antenatal anti-D prophylaxis for RhD negative women: a systematic review and economic evaluation. Health Technol Assess. 2009;13:1-126.

5. National Institute for Health and Care Excellence. Routine antenatal anti-D prophylaxis for woment who are rhesus D negative (TA156). London: National Institute for Health and Care Excellence; 2008.

6. Banch Clausen F, Steffensen R, Christiansen M, et al. Routine noninvasive prenatal screening for fetal RHD in plasma of RhD-negative pregnant women - 2 years of screening experience from Denmark. Prenat Diagn. 2014;34(10):1000-5. https://doi.org/10.1002/pd.4419.

7. Thurik FF, Ait Soussan A, Bossers B, et al. Analysis of false-positive results of fetal RHD typing in a national screening program reveals vanishing twins as potential cause for discrepancy. Prenat Diagn. 2015;35(8):754-60. https://doi. org/10.1002/pd.4600.

8. Saramago P, Yang $H$, Llewellyn A, Walker $R$, Harden M, Palmer S, Griffin S, Simmonds M. High-throughput non-invasive prenatal testing for fetal rhesus D status in RhD-negative women not known to be sensitised to the RhD antigen: a systematic review and economic evaluation. Health Technol Assess. 2018;22(13):1-172. (https://www.nice. org.uk/guidance/dg25)

9. High-throughput non-invasive prenatal testing for fetal RHD genotype: NICE Diagnostics guidance [DG25]; National Institute for Health and Care Excellence (NICE) November 2016.

10. Liberati A AD, Tetzlaff J, Mulrow C, Gøtzsche PC, loannidis JP, Clarke M, Devereaux PJ, Kleijnen J, Moher D. The PRISMA statement for reporting systematic reviews and meta-analyses of studies that evaluate healthcare interventions: explanation and elaboration. BMJ. 2009:339:b2700.

11. Whiting PF, Rutjes AW, Westwood ME, et al. QUADAS-2: a revised tool for the quality assessment of diagnostic accuracy studies. Ann Int Med. 2011; 155(8):529-36.

12. Reitsma JB, Glas AS, Rutjes AW, et al. Bivariate analysis of sensitivity and specificity produces informative summary measures in diagnostic reviews. J Clin Epidemiol. 2005;58(10):982-90. https://doi.org/10.1016/j. jclinepi.2005.02.022.

13. Rutter CM, Gatsonis CA. A hierarchical regression approach to meta-analysis of diagnostic test accuracy evaluations. Stat Med. 2001;20(19):2865-84

14. R Development Core Team. R: a language and environment for statistical computing. 2008 http://www.R-project.org.

15. Simmonds MC, Higgins JP. A general framework for the use of logistic regression models in meta-analysis. Stat Meth med Res. 2016;25(6):2858-77.

16. Chitty LS, Finning K, Wade A, et al. Diagnostic accuracy of routine antenatal determination of fetal RHD status across gestation: population based cohort study. BMJ. 2014;349:g5243. https://doi.org/10.1136/bmj.g5243.

17. Finning K, Martin P, Summers J, et al. Effect of high throughput RHD typing of fetal DNA in maternal plasma on use of anti-RhD immunoglobulin in RhD negative pregnant women: prospective feasibility study. BMJ. 2008; 336(7648):816-8. https://doi.org/10.1136/bmj.39518.463206.25.

18. Soothill PW, Finning K, Latham T, et al. Use of cffDNA to avoid administration of anti-D to pregnant women when the fetus is RhDnegative: implementation in the NHS. BJOG. 2015;122(12):1682-6. https:// doi.org/10.1111/1471-0528.13055

19. Akolekar R, Finning K, Kuppusamy R, et al. Fetal RHD genotyping in maternal plasma at 11-13 weeks of gestation. Fetal Diagn Ther. 2011;29(4):301-6. https:// doi.org/10.1159/000322959.

20. Wikman AT, Tiblad E, Karlsson A, et al. Noninvasive single-exon fetal RHD determination in a routine screening program in early pregnancy. Obstet Gynecol. 2012;120(2 Pt 1):227-34. https://doi.org/10.1097/AOG. Ob013e31825d33d9.

21. Wikman T, Tiblad E, Westgren M. Noninvasive prenatal screening for RHD: the Stockholm study. Vox Sang. 2012;103(Suppl. 1):33-4. https://doi.org/10. 1111/j.1423-0410.2012.01615-1.x.

22. Singleton BK, Green CA, Avent ND, et al. The presence of an RHD pseudogene containing a 37 base pair duplication and a nonsense mutation in Africans with the Rh D-negative blood group phenotype. Blood. 2000;95(1):12-8

23. Lun FM, Chiu RW, Chan KC, et al. Microfluidics digital PCR reveals a higher than expected fraction of fetal DNA in maternal plasma. Clin Chem. 2008, 54(10):1664-72. https://doi.org/10.1373/clinchem.2008.111385. 
24. Wang E, Batey A, Struble C, et al. Gestational age and maternal weight effects on fetal cell-free DNA in maternal plasma. Prenat Diagn. 2013;33(7): 662-6. https://doi.org/10.1002/pd.4119.

25. Grande M, Ordonez E, Cirigliano V, et al. Clinical application of midtrimester non-invasive fetal RHD genotyping and identification of RHD variants in a mixed-ethnic population. Prenat Diagn. 2013;33(2): 173-8. https://doi.org/10.1002/pd.4035.

26. Faas BH, Beckers EA, Wildoer $P$, et al. Molecular background of VS and weak C expression in blacks. Transfusion. 1997;37(1):38-44.

Ready to submit your research? Choose BMC and benefit from:

- fast, convenient online submission

- thorough peer review by experienced researchers in your field

- rapid publication on acceptance

- support for research data, including large and complex data types

- gold Open Access which fosters wider collaboration and increased citations

- maximum visibility for your research: over $100 \mathrm{M}$ website views per year

At $\mathrm{BMC}$, research is always in progress.

Learn more biomedcentral.com/submissions 